\title{
Microbiological hazards of infusion therapy
}

Edited by I. Phillips, P. D. Meers and P. F. D'ArCy. 1976. Lancaster: MTP Press Ltd. Pp. $x$ and 186. $£ 6.95$.

Published proceedings of symposia often make poor reading. At worst the papers presented are merely brought together and interspersed with snippets of discussion; at best the inevitable unevenness of subject treatment is ironed out and coherence given to the whole by the application of editorial skills. That this volume reads more like a textbook is a tribute to its editors.

A symposium, sponsored by a manufacturer of materials for infusion therapy, was held at the University of Sussex in March 1976 with the object of assembling current knowledge about the microbial insults to which patients may be subjected from defects in the manufacture and administration of infusion fluids, and about their clinical consequences. The contributions are grouped into two sections, one dealing with sources of contamination, the other with clinical problems, and each is followed by a record of the panel discussion on questions of practical detail. Critical accounts are given of the design of containers and their methods of closure, the relative ability of different bacterial species to proliferate in various kinds of fluid, and of the problems of recognising microbial contamination-both by laboratory methods, and by the clinical and epidemiological surveillance of patients receiving infusions. The various clinical syndromes, including endotoxic shock, resulting from intrinsic or extrinsic contamination are dealt with separately by different authors. There is an extensive and detailed analysis of the ways in which microbial hazards may develop during the actual administration of infusions, and the description of measures for their avoidance should prove helpful to those concerned with the hygiene of intensive therapy units.

Microbiologists and pharmacists will appreciate the convenience of a modest-sized volume that holds so much detailed information, including 279 references, in respect of a topic that is rarely the subject of a comprehensive review. The doctors who have to set up drips and the nurses who have to monitor them in use will find in it much that is directly relevant to daily practice.

J. E. M. WHITEHEAD

The generation of antibody. A new look

Edited by A. J. Cunningham. 1976. London: Academic Press. Pp. 211. $£ 7 \cdot 80$.

The mechanism of generation of antibody diversity is a central problem in immunology and one that may throw light on genetic control in other systems. The excellent review articles in this monograph illustrate the way in which problems that appear peculiarly intractable may nevertheless lend themselves to experimental approach. It is to be hoped that the present emphasis on practical research will not interfere with this process.

The first article, by Adam and Weiler, describes the remarkable properties of anti-idiotype systems and the very sophisticated regulations that they allow. It draws attention to the traditional argument that generation of diversity cannot arise by somatic mutation during ontogeny because the cells that mutate early would comprise a disproportionately high fraction of the final lymphocyte population. The authors show, first by intuitive arguments and then by mathematical deduction, that the occurrence of anti-idiotype interactions between lymphocyte populations, of the type described in Jerne's network hypothesis, would overcome the problem. Historically, the molecular biology of the bacteriophage was developed by physicists and it is heartening to see that immunology is now being influenced by modes of thinking usually associated with theoretical physics.

Cunningham develops Burnet's idea that the generation of antibody diversity occurs after antigenic stimulation as well as during ontogeny, while Williams and Fitzmaurice discuss the implication of the joining up of $\mathrm{V}$ and $\mathrm{C}$ genes in terms of the behaviour of the DNA of the chromosome.

This book should encourage immunologists to look at their problems more broadly and to ask whether these may not throw light on other areas of biology. It is not an easy book to 